\title{
A Novel Method for L Band SAR Image Segmentation Based on Pulse Coupled Neural Network
}

\author{
Harwikaryaa $^{\mathrm{a}, *}$, Sabar Rudiarto ${ }^{\mathrm{a}}$, Glorin Sebastian ${ }^{\mathrm{b}}$ \\ ${ }^{a}$ Informatic Departmentst, Universitas Mercu Buana, Jl.Meruya Selatan No 1, Jakarta 11650, Indonesia \\ ${ }^{b}$ Georgia Institute of Technology, United State Of America \\ ${ }^{1}$ harwikarya@mercubuana.ac.id*; ${ }^{2}$ sabar.rudiarto@mercubuana.ac.id \\ * corresponding author
}

\section{ARTICLE INFO}

Article history:

Received 24 March 2020

Revised 10 May 2020

Accepted 20 June 2020

Keywords:

Segmentation

Pulse Couple Neural Network

Gray Level Co-occurence Matrix

Synthetic Apperture Radar

\begin{abstract}
Pulse Coupled Neural Network (PCNN) is claimed as a third generation neural network. PCNN has wide purpose in image processing such as segmentation, feature extraction, sharpening etc. Not like another neural network architecture, PCNN do not need training. The only weaknes point of PCNN is parameter tune due to seven parameters in its five equations. In this research we proposed a novel method for segmentation based on modified PCNN. In order to evaluate the proposed method, we processed L Band Multipolarisation Synthetic Apperture Radar Image. The Results showed all area extracted both by using PCNN and ICM-PCNN from the SAR image are match to the groundtruth. There fore the proposed method is work properly.
\end{abstract}

Copyright (๑) 2017 International Journal of Artificial Intelegence Research. All rights reserved.

\section{Introduction}

The researchs on image segmentation have been proposed in this decade. Segmentation is a mechanism of partiting image into homogeneous and specifik regions. Segmentation is fundamental step in image analysis, pattern recognition and computer vision. The results of image segmentation is very important as an input to image classification. Segmentation algorithm which had been proposed are region based [1], edge based [2], threshold base [3], fuzzy theory [4] and neural network base [5].

Region based algorithm consist of region splitting and merging method and region growing method. Region splitting and merging is formally based on quadtree data theory. In this algorithm image is divided or splitted into some set image which is arbritrary and disconnected regions, and these regions would be merge or split to meet the condition of segmentation. One of the improvement in region based algorithm had been published [6].

Edge based algorithm detecs edges or sharp variation in intensity of pixels. This sharp variation indicates the boundary of target. The result of segmentation depend on the accuracy of detection of sharp variation. The novel research on edge based by using the bacterial foraging had been proposed [7].

Artificial Neural Network mostly works or acts as a human brain. The important organs in human brain are dendrit, neuron and synapse. All these organ are mimiced in form of program in certain programming language. Many kinds architecture of antificial neural network had been used in many domain of resesarch, such as back propagation neural network, radial basis function (RBF) neural network, pulse coupled neural network (PCNN). Pulse Coupled Neural Network (PCNN) is claimed as third generation neural network, it is a mimic of the vision system or cortex of mamalia [8]. The mimic is formed in mathematical description, this concept was first put forward by Eckhorn then corrected by Johnson using a cortical concept so that it can be used in the field of image processing [9]. The advantage of PCNN is it can opearate without any training needed but it has the problem; all parameters in the equation have to be tuned [10]. Many researchers have used PCNNs in research on image segmentation, eliminating noise, edge 
detection, feature extraction and pattern recognition, enhancing image quality, and fusion or merging images [11]. Image segmentation process can be done on color image objects, gray level images, for example gray and textured radar images. This segmentation research is the first step in the research of land cover classification on radar imagery [12] [13] [14] [15]. In edge detection studies, PCNN can be used to see the boundaries between objects and backgrounds in gray level images, the results of the study are quite good compared to the Sobel edge detection method, Cany Method, Laplacian Gaussian method [16]. Research on feature extraction and pattern recognition, PCNN is rarely used, but from existing literature searches have been used for face recognition of several people in one color image and the results are reported to be good [17]. Some researchers claim that PCNN is a third generation artificial neural network, the strength of this PCNN is that it does not require learning and does not require examples of images to be processed, but on the other hand has weaknesses which are seven parameters that must be optimized, this is something that is not easy to do [18]. To facilitate parameter optimization some researcher modified the equation. The way to modified the equation could be grouped in to three mecanism, intersecting cortical model (ICM), linking unit PCNN model (UL-PCNN) and spiking cortical model (SCM).

Here, we propose a novel method for segmentation based on ICM- PCNN. In this method, we attempt to build a new method by taking account the texture features of each input SAR image and the dynamic properties of two dimension neuron layers to determine the proper segmented images.

\section{Related works}

\section{A. Intersecting Cortical Model}

In the intersecting cortical model (ICM) method, PCNN equation from five equations are simplified to three equations. Modifications eliminate linking or equation number 2 and internal modulation or number 3 without losing the nature of PCNN. ICM is the result from the mammalians' research on the visual cortex. The activities of cortex or visual system could be simulated in two- dimension neurons model. ICM uses the feeding factor in equation (1). In this equation, $S_{i, j}$ is the value of the pixel in the observation window, it represents of mammalian optic system. The two-dimension of simplified structure of ICM is shown in Fig. 1 [19]. Single neuron cell receives the output of neighbour, $\mathrm{Y}_{k, 1}$ coupled with the vector $\mathrm{W}$ or weighting function. Feeding is compared to the threshold $\Theta_{i, j}$ as a basic of output state $Y_{i, j}$

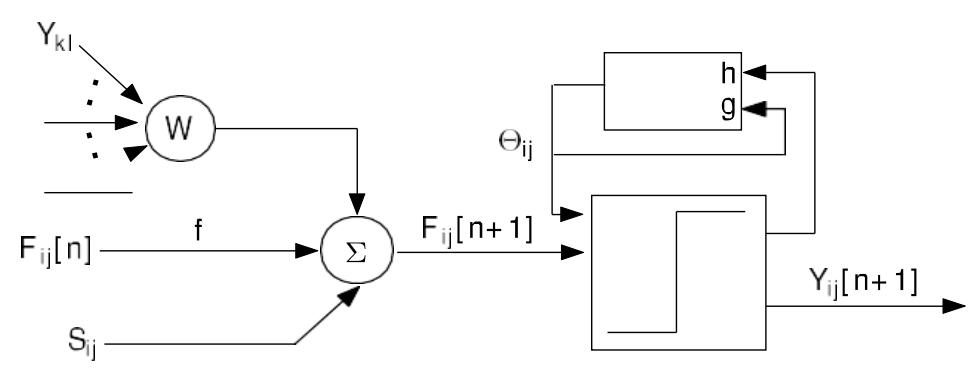

Fig.1. Neuron Cell

In image processing a pixel corresponds to one neuron cell. In the ICM network, the state oscillators of all the neurons are represented by a $2 \mathrm{D}$ array or Feeding as $\mathrm{F}_{\mathrm{i}, \mathrm{j}}$ and the threshold oscillators of all the neurons by a $2 \mathrm{D}$ array or $\Theta_{\mathrm{i}, \mathrm{j}}$ as mentioned before.

The equations of ICM-PCNN as follows:

$$
\begin{array}{r}
F_{i j}[n]=e^{-\alpha_{F}} F_{i j}[n-1]+S_{i j}+V_{F} \sum_{k l} m_{i j k l} Y_{k l}[n-1] \\
\Theta_{i j}[n]=e^{-\alpha_{\Theta}} \Theta_{i j}[n-1]+V_{\Theta} Y_{i j}[n-1] \\
Y_{i j}[n]=\left\{\begin{array}{l}
\left.1, \text { if } U_{i j}[n]\right\rangle \Theta_{i j}[n] \\
0, \text { otherwise }
\end{array}\right.
\end{array}
$$


In which, the subscript $i$ and $j$ is the coordinate of each pixel and $\mathrm{Y}_{\mathrm{i}, \mathrm{j}}$ is the output value which is either 1 or 0 . The ICM-PCNN could process the input image to extract the area in the image. Each area represent by white pixels as the output of certain number of iteration indicate by equation (3). In thsi equation one cell neuron only has two outputs 1 or white pixel and 0 or black pixel. The ICM-PCNN has three parameters such as $\alpha_{F}, \alpha_{\Theta}$ and $V_{F}$. Therefore ICM-PCNN works faster than primitive PCNN which has five equations and 7 parameters.

ICM-PCNN has been used for image segmentation of aircraft on the runway and vehicles on the highway. The parameters in this works are $\alpha_{F}=0.9, \alpha_{\Theta}=0.8$ and $V_{F}=20$. The results are reported good [20]. Guangzhu at.al used ICM-PCNN to study the iris that will recognize someone from the iris character. The value of parameters in this research are $\alpha_{F}=0.08, \alpha_{\Theta}=0.32$ and $V_{F}=2$. The results of the study were reported well [19]. Mikrut at.al proposed ICM-PCNN to analyse air photogrammetric. The highway, buildings and others in these aerial photographs can could be segmented properly [21]. In [22] ICM-PCNN is used to view ultrasound images on the chest so that it will help radiologists to distinguish healthy and injured tissue and the results is reported good. Ahmed at.al have proposed ICM-PCNN for face recognition on colored optical images. The parameter used are $\alpha_{F}=0.9, \alpha_{\Theta}=0.8$ and $V_{F}=20$. The accuracy in face recognition in this works is more than 95\%. [20]. Another study in ICM-PCNN was proposed by Chai Ling at.al and . This study focus on noise removal, especially from an image that contains a lot of noise. ICM-PCNN was developed into improved ICM called IICM [23]. The application of ICMPCNN in medical world is used to segment brain image [24]. This works attempt to segment the normal cell and cancer cell in the Nuclear Magnetic Resonance Image.

\section{B. Segmentation}

Segmentation based on texture has difficulties, especially when the image is in complex textured. Despites the difficulties, segmentation has an important role in computer vision. For two decades segmentation has an important role as an important technique in image processing especially in computer vision. Segmentation can be operated in biomedical image analysis [25] , industrial inspection [26] and satellite image analysis [27]. Textures has complexity in form or composition of pixels, it is difficult to provide a universal definition of textures [28]. An automated image segmentation in blood vessel was proposed in Ref [29], this method based on PCNN and 2D Otsu algorithm. PCNN was used to segment images in different ilumination [30], the results was reported good. In the medical imaging, PCNN was used to segment magnetic resonance image from the brain as mentioned before in Ref [24]. PCNN could made good contrast between normal cell and cancer cell. In this research PCNN was combined with Back Propagation Neural Network. The results could help the physician to analyse the MR images fastly.

The digital image with a size of $\mathrm{M}^{*} \mathrm{~N}$ and a grayscale value of $\mathrm{L}$, could be considered as a twodimensional matrix; $\mathrm{M}$ is the row and $\mathrm{N}$ is the collumn. The values of the pixels varie from 0 to L. When image segmentation is processing, the matrix elements and PCNN model neurons can be put into one-to-one correspondence. The pixel value $\mathrm{I}_{\mathrm{ij}}$ corresponds to the neurons' input $\mathrm{Sij}$ on the corresponding position. For the first iteration the initial state of the neurons was set to 0 . Following the first iteration, internal activity $\mathrm{U}_{\mathrm{ij}}$ is equal to the neurons' input $\mathrm{Sij}$, all the neurons threshold attenuated from the initial value, when a neuron's threshold attenuated till smaller or equal to the correspond $U_{i j}$, this neuron was fired(natural fired), and output a pulse $Y_{i j}=1$, simultaneously, the threshold $\Theta_{\mathrm{ij}}$ of this neuron sharply increased, the pulse output stopped. Then $\Theta_{\mathrm{ij}}$ began to attenuate, when $\Theta_{\mathrm{ij}}$ attenuated till smaller or equal to correspond $U_{\mathrm{ij}}$ again, pulsegenerated again. In every several cycles the neurons produce an output sequence, which contains information describing the area, boundary, texture and other characteristics of the digital image. In other word PCNN can do segmentation in digital image.

In general, all textured images mostly have two general characteristics, homogeneity and nonhomogeneity. The examples of textures are showed in Figure 1, taken from the Brodatz album. This figure showed homogenous, weakly homogenous and in homogenous texture. Weakly homogeneous and in homogeneous textured images are interested object in image processing and pattern recognition. 


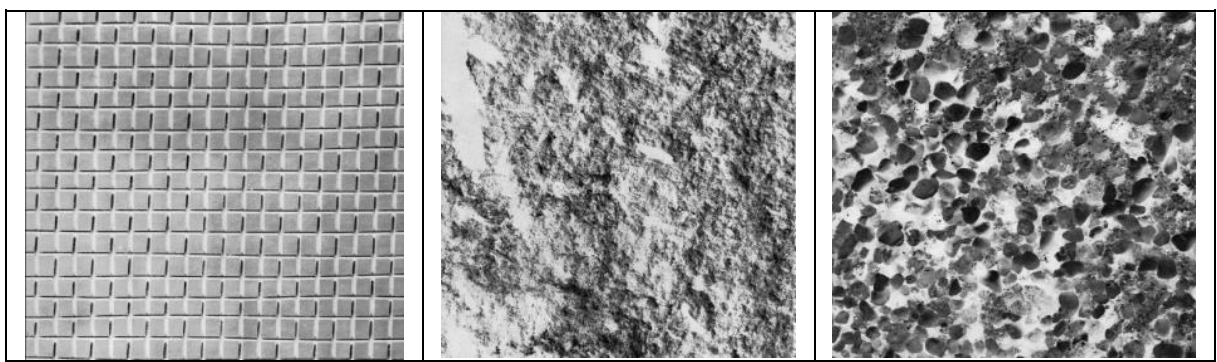

Fig 2. Homogenous, weakly homogenous and in homogenous texture

\section{Proposed Method}

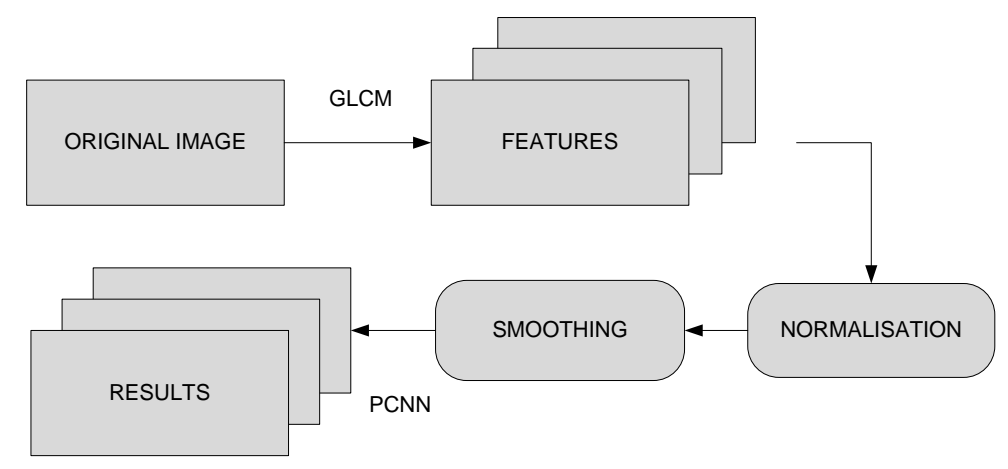

Fig. 3. Proposed Method

The proposed method is depicted in Figure2. Original image is Synthetic Aperture Radar Image, L band full polarization; Horizontal- Horizontal (L-HH), Horizontal Vertical (L-HV), Vertical Vertical (L-VV), Vertical -Horizontal (L-VH). These images are acquired by using ESAR System. Images contain water area, bare land and forest from certain area in Penajam East Kalimantan.

First step the original images are extracted by using Grey Level Co-occurrence Matrix (GLCM) The results are texture's features, we limited only use three features, such as correlation, dissimilarity and angular second moment or second moment.

Second step the value of all features is normalized to get the value of pixel in grey level image from the features, the range of the pixels value is between 0 to 255 .

Third step is smoothing, all pixel value in a certain dimension of window are smoothed with the aim to avoid the very high differences among the pixels.

The last step is segmentation by using Pulse Coupled Neural Network (PCNN). In this segmentation we use primitive PCNN and ICM-PCNN.

\section{Results and Discussion}

The object of the research as mention before are SAR images in L Band showed in Figure 4 which contains L-HH, L-HV, L-VV and L-VH. These SAR images are in homogeneous textured. Refer to ground truth, this image has three area, housing including the street, forest and water. The dark or black area are water consist of river and swamps, the light area are housing and street and in between there are the forest. L-VV has light area in the river, it is the wave, the other polarization has not light are or wave.

In order to verify the proposed method this research use Matlab 7.1 to extract the features of the SAR image and other process such as normalization, smoothing and segmentation by using Pulse Coupled Neural Network (PCNN). 


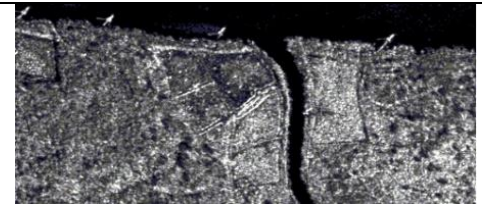

(a)

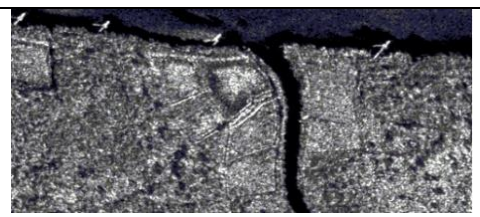

(c)

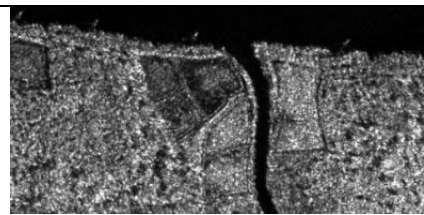

(b)

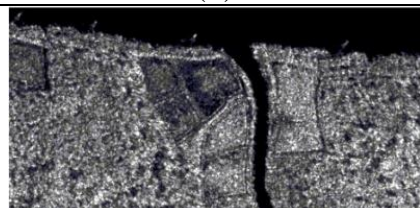

(d)

Fig. 4. L-Band SAR Image, (a) L-HH, (b)L-HV, (c)L-VV and (d) L-VH

\section{A. Gray Level Co-occence Matrix Features}

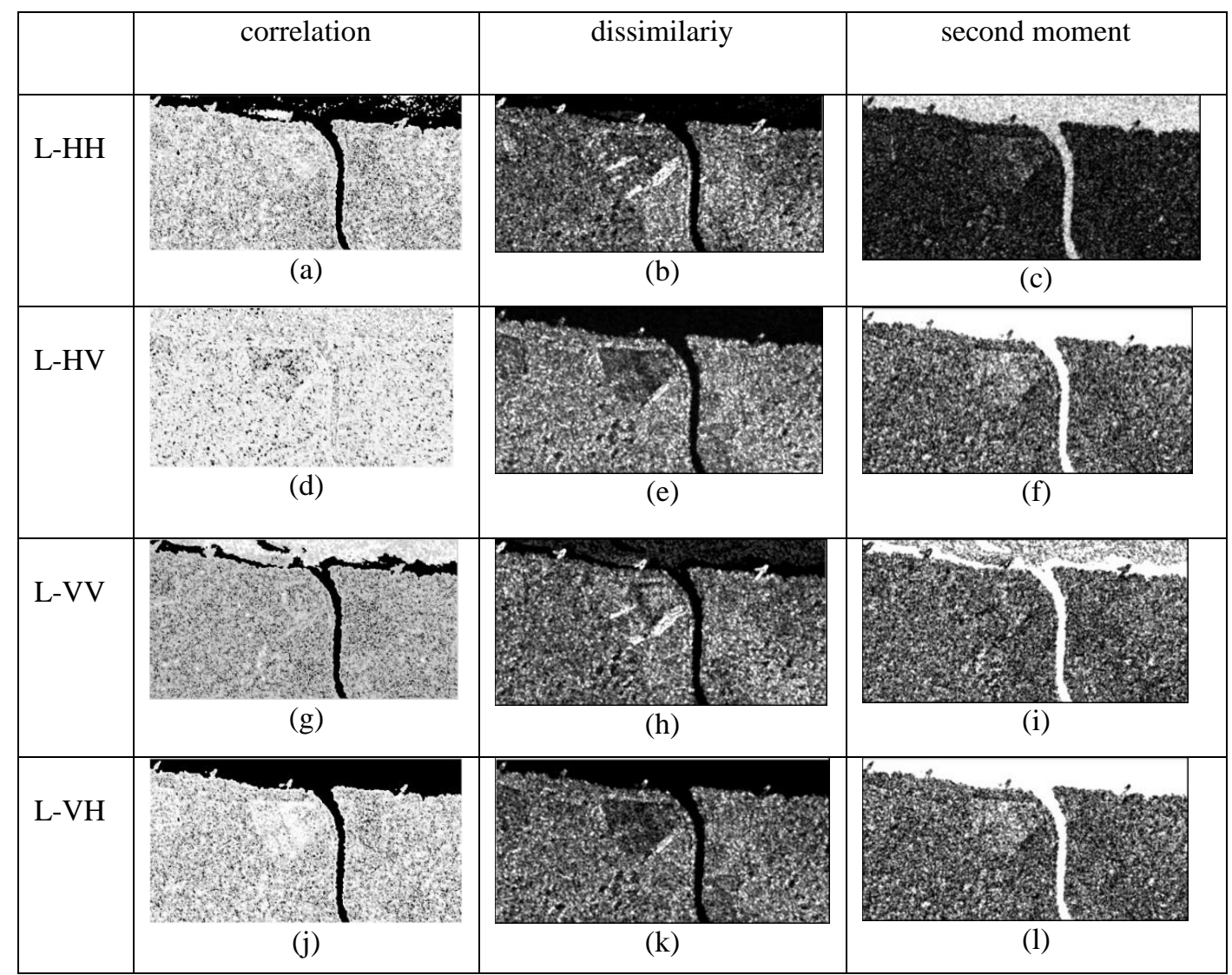

Fig. 5. Three features : correlation, dissimilarity and second moment.

Figure 5 showed the results of features extraction, it is focused on three features such as: correlation, dissimilarity and second moment. Correlation represents the open area or bare land refer to the ground truth. L-VH is the best among the three others polarisation. In L-HH, figure 5 (a), the water area has the white pixel, therefore there are an overlap with other area, it could be an open area or bare land. In L-HV, figure 5(d), correlation contain almost white pixels, there are an overlap among all area. In L-VV, figure $5(\mathrm{~g})$, white pixels appear in water area, overlap is dominant in water area. From all four polarisation, correlation in L-VH, figure 5(j), is the best reperesentative of bare land. Therefore this result will be processed in next step.

Dissimilarty represents the forest area, refer to the ground truth, these forest area overlap with the housing which stand in the river bank. L-HH, figur 5(b), showed the forest and bare land in the 
white pixels. The housing and the small port for the boat could be seen clearly, but there are some white pixels in the water area, it means there are overlap eventhoungh only very small white pixels. In L-HV, figure 5 e), there are overlap in bare land, but no overlap in water area. Water area are almost in black pixels. The port and housing somewhat less than in L-HH. In L-VV, figure 5(i), the forest, housing and port are overlap one and others. In the water area there are a couple of white pixels which not repreent the forest and housing. The last in $\mathrm{L}-\mathrm{VH}$, figure $5(\mathrm{k})$, there are no overlap in water area, the water contains almost black pixels. The forest, housing and the port are al in white pixels. Therefore L-HH and L-VH could be considered or taken count as a forest and housing area. In this SAR image housing and forest have almost same brightness due to the high of the forest which contain sawit tree, not a high wood forest.

Angular second moment or second moment represents the water area, in this SAR images water area consist of river and swamp. L-HH, figure 5(c), showed the water area in white pixels but there are some black pixels which indicate not water area. In L-HV, figure 5 (f), the water area are clearly in white pixels both the rivers and swamps.

The results of L-VV, figure 5 (i), contains overlap both in water area and other area. In water area there are some black pixels and in bare land and forest there are some white pixels. The last result depicted in figure 5 (1), no over lap, water area are clear. Therefore the best result for the water area is in $\mathrm{L}-\mathrm{VH}$ in second moment feature.

The all result of features extraction give lots of information and interpretation. The water area is best represented by $\mathrm{L}-\mathrm{VH}$ in second moment feature, bare land is given by $\mathrm{L}-\mathrm{VH}$ in correlation feature and forest is represented by $\mathrm{L}-\mathrm{VH}$ in dissimilarity feature. Therefore all area could be extracted only in L-VH SAR image. Overlap in the results gave other interpretation, for example overlap in water area in L-HH, figure 5(c) and L-VV, figure 5(i), could be interpretated as a wave on the river due to reflexion caught by the sensor. It need another resesarch to confirm this interpretation by using the calibrator of sea wave height.

\section{B. Pulse Coupled Neural Network (PCNN)}

All features are processed by primitive PCNN in form of five equations. Each polarisation gives a different results. The all parameters of PCNN are showed in Table 1.

Table 1. Parameters of PCNN

\begin{tabular}{|c|c|c|}
\hline No. & Parameter & Range \\
\hline 1 & $\alpha_{\mathrm{F}}$ & $0.1-1$ \\
\hline 2 & $\alpha_{\mathrm{L}}$ & $0.1-10$ \\
\hline 3 & $\mathrm{~V}_{\mathrm{F}}$ & $1-10$ \\
\hline 4 & $\mathrm{~V}_{\mathrm{L}}$ & $1-10$ \\
\hline 5 & $\alpha_{\Theta}$ & $0.1-1$ \\
\hline 6 & $\beta$ & $0.1-1$ \\
\hline 7 & $\mathrm{~V}_{\Theta}$ & $0-100$ \\
\hline
\end{tabular}

The results are depicted in Fig. 6. Despite the best results of feature extraction is L-VH, in this step all polarisation are processed by iterating into primitive PCNN.

All features are processed by primitive PCNN in form of five equations. Each polarisation gives a different results. The all parameters of PCNN are showed in Table 1.

In L-VH, figure 6(j), correlation feature represent the bare land area. The water area are totally in black pixels, no over lap in water area. In L-VV, figure $6(\mathrm{~g})$, there are over lap in water area, it is indicated by spreaded white pixels in water area. In L-HV over lap is occured in bare land indicating by white pixels. In L-HH, figure 6(a) there are over lap in all area, the white pixels did not represent any area. Correlation feature in all polarisation indicated bare land area, certain 
polarisation has certain white pixels, from minimum to maximum number. In ground truth the bare land has grass and in certain area there are shrub and underbrush. Bare land are usually prepared for the sawit tree to produce palm oil. In this works GLCM features only assigned to L band SAR image, the numbers of white pixels depend on the topography of the terrain that had been acquired.

\begin{tabular}{|c|c|c|c|}
\hline & correlation & dissimilariy & second moment \\
\hline \multirow[t]{2}{*}{ L-HH } & & & \\
\hline & (a) & (b) & (c) \\
\hline \multirow[t]{2}{*}{ L-HV } & & & \\
\hline & (d) & (e) & (f) \\
\hline \multirow[t]{2}{*}{ L-VV } & & & \\
\hline & (g) & (h) & (i) \\
\hline \multirow[t]{2}{*}{ L-VH } & & & \\
\hline & (j) & (k) & (l) \\
\hline
\end{tabular}

Fig. 6. The results of primitive PCNN.

\section{Interseting Cortical Model Pulse Coupled Neural Network (ICM-PCNN)}

The results of ICM - PCNN are depicted in Figure 7. Each polarisation gives the diffrerent result similar to primitive PCNN. We directly focus on L-VH polarisation, all features represented all area such as bare land, forest and water.

Table 1. Parameters of ICM-PCNN

\begin{tabular}{|c|c|c|}
\hline No. & Parameter & Range \\
\hline 1 & $\alpha_{\mathrm{F}}$ & $0.1-10$ \\
\hline 2 & $\mathrm{~V}_{\mathrm{F}}$ & $1-10$ \\
\hline 3 & $\alpha_{\Theta}$ & $0.1-1$ \\
\hline
\end{tabular}

First analyse is on correlation in figure 7(j), it is good because there are no over lap, the water area are totally in black pixels and the forest either. Bare land is clearly white and exactly similar to the ground truth. If it compare to another result, $\mathrm{L}-\mathrm{VV}$ in figure $7(\mathrm{~g})$ and $\mathrm{L}-\mathrm{HV}$ in figure $7(\mathrm{~d})$ there are overlap and no bare land inside the picture since the pixels are mostly in black. L-HH in figure 7(a) contains over lap both in water and bare land and forest. It can be concluded that correlation in L-VH represented bare land and it is the best results among L-VV, LHH and L-HV. 
Next features is dissimilarity, refer to the previous results we could see in L-VH in figure $7(\mathrm{k})$ is verry good. Compare to another polarisation, all results are verry similar, the contradiction only in small part in bare land in South East direction. L-HH in figure 7(b) and L-VV in figure 7(h) the bare land in the direction mentioned before are widen, this is un match to the ground truth. In L-HV the small part of forest in North East direction are in black pixels, but the results in L-HV is very similar to L-HV.

\begin{tabular}{|c|c|c|c|}
\hline & correlation & dissimilariy & second momen \\
\hline L-HH & (a) & (b) & $\begin{array}{l}\text { (c) } \\
\text { (c) }\end{array}$ \\
\hline L-HV & (d) & (e) & (f) \\
\hline L-VV & (g) & (h) & (i) \\
\hline L-VH & (j) & (k) & (l) \\
\hline
\end{tabular}

Fig 7. The results of ICM-PCNN

Second moment feature represents water area due to the results of all polarisation depicted in figure 7(c), 7(f), 7(i) and 7(1). In all polarisation water area are indicated by white pixels and another area are in black pixels. In L-HH there are overlap in water area indicated by a few black pixels but over all the water area are dominant. L-HV showed the good contrast between water area and another area, water area in white pixels and another area in black pixels, these black pixels are the darkest among the black pixels in another polarisation. L-VV has a couple of black pixels in water area indicates there are overlap. $\mathrm{L}-\mathrm{VH}$ is the best among another polarisation due to the results of iteration by PCNN. There are no over lap both in water area and another area. Therefore $\mathrm{L}-\mathrm{VH}$ is the best for all area such as bare land, forest and water.

The final step in this method all feature is overlaid to form the entire area in L Band SAR image based on primitive PCNN and ICM-PCNN. The entire segmented area is depicted in figure 8.

PCNN and ICM-PCNN gave the good results in segmentation of L band SAR image, figure 7 showed there are no significant contrast. In figure 8(a) and 8(b) water area, blue pixels, are similar or quasi same, clear and no overlap. Bare land, in red pixels figure 7(b) is wider than red pixels in figure 7(a), forest, green pixels, in 8(a) is wider than green pixels in figure 8(b). Over all the result of PCNN and ICM-PCNN are good. We have to count the number of each pixels to get the exact information about the number of each pixels and to check or refer to the ground truth. 


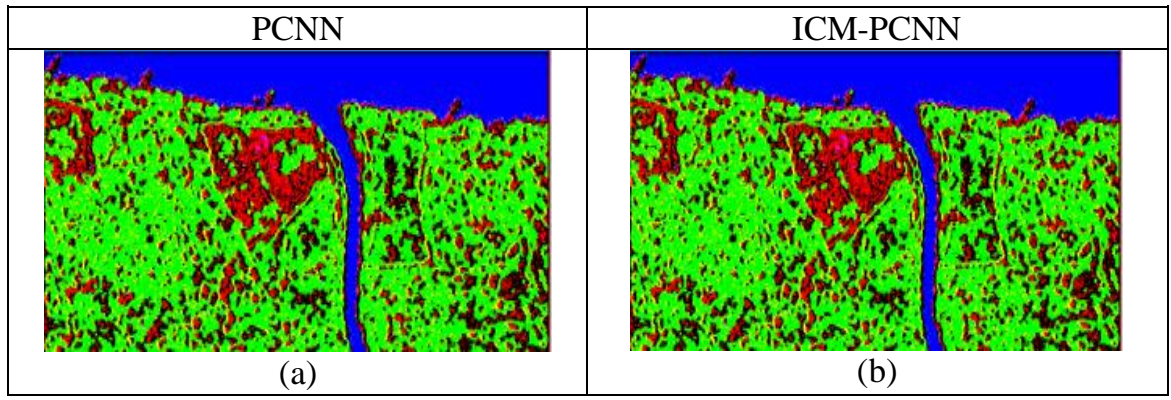

Fig. 8. The results of PCNN and ICM-PCNN

\section{Conclussion}

In this paper we proposed novel methodology to segment the textured images, in this research we use L band multi polarization SAR image. The GLCM features extracted from all polarisation such as correlation, dissimilarity and angular second moment explaine the certain area on acquired terrain. L-VH give the best results among all polarisation which is correlation describes the bare land, dissimilarity describes forest and housing and angular second moment describes water area. The processed of these features by using PCNN and ICM-PCNN give the good results. All area are are clear segmented both using PCNN and ICM-PCNN, there are no significant contrast. Bare land in ICM-PCNN is wider than bare land in PCNN and the forest in PCNN is wider than the forest in ICM_PCNN. The results have to compare to ground truh for fair conclusion. It should be noted that this new method proposed gives good results. Both the combination of GLCM and primitive PCNN and GLCM and ICM-PCNN work good. The future work should be focused on another textured images such as in radiology, radiography, NMR and others to confirm the though of proposed method and to have the computation time between primitive PCNN and ICM-PCNN by using big two dimensions textured image.

\section{References}

[1] H. Yu, F. He, and Y. Pan, "A novel region-based active contour model via local patch similarity measure for image segmentation," Multimed. Tools Appl., 2018.

[2] "IMAGE SEGMENTATION BY USING EDGE DETECTION," Int. J. Comput. Sci. Eng., 2010.

[3] K. Bhargavi and S. Jyothi, "A Survey on Threshold Based Segmentation Technique in Image Processing," Int. J. Innov. Res. Dev., 2014.

[4] I. Soesanti, A. Susanto, T. S. Widodo, and M. Tjokronagoro, "Optimized Fuzzy Logic Based Segmentation for Abnormal MRI Brain Images Analysis," Int. J. Comput. Sci. Issues, 2011.

[5] K. Jiao and Z. Pan, "A Novel Method for Image Segmentation Based on Simplified Pulse Coupled Neural Network and Gbest Led Gravitational Search Algorithm," IEEE Access, 2019.

[6] Y. Chen and C. Han, "A modified region growing algorithm for multi-colored image object segmentation," Chinese Opt. Lett., 2007.

[7] Y. Pan, T. Zhou, and Y. Xi, "Bacterial foraging based edge detection for cell image segmentation," in Proceedings of the Annual International Conference of the IEEE Engineering in Medicine and Biology Society, EMBS, 2015.

[8] R. Eckhorn, H. J. Reitboeck, M. Arndt, and P. Dicke, "Feature Linking via Synchronization among Distributed Assemblies: Simulations of Results from Cat Visual Cortex," Neural Comput., 1990.

[9] Y. Chen, S. K. Park, Y. Ma, and R. Ala, "A new automatic parameter setting method of a simplified PCNN for image segmentation," IEEE Trans. Neural Networks, 2011.

[10] N. Yang, H. J. Chen, Y. F. Li, and X. L. Hao, "Coupled parameter optimization of PCNN model and vehicle image segmentation," Jiaotong Yunshu Xitong Gongcheng $Y u$ 
Xinxi/Journal Transp. Syst. Eng. Inf. Technol., 2012.

[11] W. Nianyi, M. Yide, and Z. Kun, "Development of PCNN research and its application in voice recognition," J. Converg. Inf. Technol., 2012.

[12] harwikarya, "Klasifikasi Citra Sinthetic Apperture Radar Berdasarkan Pulse Coupled Neural Networks Dikombinasikan Dengan Ciri Tekstur," Disertasi. Fak. Ilmu Komput. Univ. Indones., 2009.

[13] Harwikarya, "Classification of SAR Images Based on Pulse Coupled Neural Networks: Case on L-VH Band and C-VH Band," J. FIFO, vol. IV/N0.4.

[14] J. Karvonen, "Simplified Pulse-Coupled Neural Network based sea-ice classifier with graphical interactive training," in International Geoscience and Remote Sensing Symposium (IGARSS), 2000.

[15] J. A. Karvonen, "Baltic sea ice SAR segmentation and classification using modified pulsecoupled neural networks," IEEE Trans. Geosci. Remote Sens., 2004.

[16] X. Deng and Y. Ma, "PCNN model analysis and its automatic parameters determination in image segmentation and edge detection," Chinese J. Electron., 2014.

[17] H.-Y. LI, R. ZONG, and D. XU, "Color Face Detection Based on PCNN Time Signature(SOFT COMPUTING METHODOLOGIES AND ITS APPLICATIONS)," Biomed. fuzzy Hum. Sci. Off. J. Biomed. Fuzzy Syst. Assoc., 2011.

[18] H.-R. Ma and X.-W. Cheng, "Automatic Image Segmentation with PCNN Algorithm Based on Grayscale Correlation," Int. J. Signal Process. Image Process. Pattern Recognit., 2014.

[19] G. Xu, Z. Zhang, and Y. Ma, "A novel method for iris feature extraction based on intersecting cortical model network," J. Appl. Math. Comput., 2008.

[20] A. G. Mahgoub, A. A. Ebeid, H. E. D. M. Abdel-Baky, and E. S. A. El-Badawy, "An Intersecting Cortical Model based framework for human face recognition," in WMSCI 2007 - The 11th World Multi-Conference on Systemics, Cybernetics and Informatics, Jointly with the 13th International Conference on Information Systems Analysis and Synthesis, ISAS 2007 - Proc., 2007, vol. 5, pp. 126-130.

[21] E. R. Sensing, "NEURAL NETWORKS IN THE AUTOMATION OF PHOTOGRAMMETRIC," vol. 1988, 1992.

[22] E. Aceves and W. Gómez, "Breast ultrasound segmentation using evolutionary pulsecoupled neural networks," in XXIII Brazilian Congress on Biomedical Engineering, 2012, pp. 922-926.

[23] L. Chai, "Adaptive image de-noising algorithm in intersecting cortical model," $J$. Multimed., 2013.

[24] M. Monica Subashini and S. K. Sahoo, "Brain MR image segmentation for tumor detection using artificial neural networks," Int. J. Eng. Technol., 2013.

[25] S. D. Yanowitz and A. M. Bruckstein, "A new method for image segmentation," Comput. Vision, Graph. Image Process., vol. 46, n, pp. 82-95, 1989.

[26] J. Kittler and J. Illingworth, "Minimum error thresholding," Pattern Recognit., 1986.

[27] J. C. Bezdek, L. O. Hall, and L. P. Clarke, "Review of MR image segmentation techniques using pattern recognition," Med. Phys., 1993.

[28] C. K. Chow and T. Kaneko, "Automatic boundary detection of the left ventricle from cineangiograms," Comput. Biomed. Res., 1972.

[29] C. Yao and H. J. Chen, "Automated retinal blood vessels segmentation based on simplified PCNN and fast 2D-Otsu algorithm," J. Cent. South Univ. Technol. (English Ed., 2009.

[30] J. L. Dansong Cheng, Wei Zhao, Xianglong Tang, "Image Segmentation Based on Pulse Coupled Neural Network," Proc. 11th Jt. Conf. Inf. Sci., 2009. 